\title{
New Fused Quinoxalines : Synthesis and Reactions of Pyrimidothienoquinoxaline and Oxadizolylthienoquinoxalines
}

\author{
Osama S. Moustafa," Mahmoud Z. A. Badr, and Talaat I. El-Emary \\ Chemistry Department, Faculty of Science, Assiat Liniversity. Assiat 7/516, Egypt \\ Received November 22. 2001
}

\begin{abstract}
Diazotization of 3-amino-2-ethoxycarbonylthieno[2,3-b]quinoxaline $\mathbf{1}$ gave the diazonium salt 2 which was reacted with $\mathrm{SO}_{2}$ and $\mathrm{N}$-methylaniline to give sulfamoylquinoxaline derivatives $3-5$. Imidazothienoquinoxaline 8 was obtained from the reaction of carboxylic acid hydrazide 6 with nitrous acid and followed by boilling the carboazide 7 in dry xylene. $\Lambda \mathrm{lso}$, compound 6 react with $\mathrm{CI}(\mathrm{OEt})$ : to give aminopyrimidine 9 which was reacted with arylidene malonodinitrile, furfural and/or dimethoxy-tetrahydrofuran to afford compounds $\mathbf{1 0}, 11$ andior 12 respectively. Refluxing of 6 with $\mathrm{CS}_{2}$ gave oxadiazolylthienoquinoxaline 13, reaction of 13 with hyrazine hydrate, $\mathrm{ClI}(\mathrm{OEt})_{3}$, nitrous acid, $\mathrm{CS}_{2}$ and $\alpha$-halocompounds to give 14-19.
\end{abstract}

Keywords : Synthesis, Diazotization, Heterocycles, Pyrimidothienoquinoxalines.

\section{Introduction}

Among the wide variety of quinoxaline derivatives that have been explored for developing pharmaceutically important molecules for examples, imidazoquinoxalines ribonucleosides as linear of antiviral,' pyrazoloquinoxaline showed a relatively high antibacterial activity wherien MIC valuc was $25 \mu \mathrm{g} / \mathrm{mL}$ against Bacillus Licheniformis and Cellulomomas Sp.. ${ }^{2}$ quinoxaline-1,4-di- $N$-oxides for treatment of 1uberculosis," pyrimido|4.5-b|quinoxaline used as anti-hypertensive and blood platelet antiaggregating agents, ${ }^{4}$ also some quinoxaline derivalives have a cylotoxic eflects on human cancer cell lines. ${ }^{50}$ commercially impotant as agrochemicals. ${ }^{7}$ herbicides, ${ }^{8}$ hypoxic-cytoxic agents, ${ }^{6}$ antivirus (I lepatitis B), "1" antimicrobial. " and amcbicides, ${ }^{12}$ we are taking all the above benelits into consideration and in continuation of our work in synthesis of fused heterocyclic rings with quinoxaline moicty. ${ }^{1 \hat{i}-19}$

\section{Results and Discussion}

In this work we aimed to synthesize some dilerent hetero- cycles systems fused with thieno[2,3-b]quinoxaline hoping that they may be highly biological activity. The reaction of 3-amino-2-clhoxycarbonylthieno|2,3-b|quinoxaline $1^{15}$ with nitrous acid and then sullur dioxide and cupric chloride in acetic acid gave the 2-cthoxycarbonylthicno|2,3-b|quinoxaline-3-sulionylchloride 3 via the diazonium salt 2 . $^{\text {Is }}$ The reaction of 3 with $N$-methylaniline alforded the 3 -sulfamoylthienoquinoxaline 4, whose hydrolysis provided the 3sulfamoyl-2-carboxylic acid 5 (Scheme 1).

3-^mino-2-1hienol2,3-b|quinoxaline-2-carboxylic acid hydrazide 6 was obtained by refluxing the coressponding ethyl ester 1 in ethanolic hydrazine hydrate ${ }^{14}$ which was reacled with nitrous acid $\left(\mathrm{NaNO}_{2} / \Lambda \mathrm{COI}\right)$ ) 10 produce the corresponding 3-amino-2-carboazide derivative 7, which underwent Curtius rearrengment when relluxed in dry xylene to the imidazothienol2,3-b|quinoxalinone 8 (Scheme 2).

The reaction of carohydrazide 6 with tricthyl orthoformate in ethanol in the presence of catalytic amount of acetic acid led to the formation of 3-aminopyrimidothieno|2.3-b|quinoxalinone 9, which was subjected to Michacl reaction when reacted with benzylidene malonodinitrile in ethanol in the presence of a lew drops of piperidine to give the pyrida-

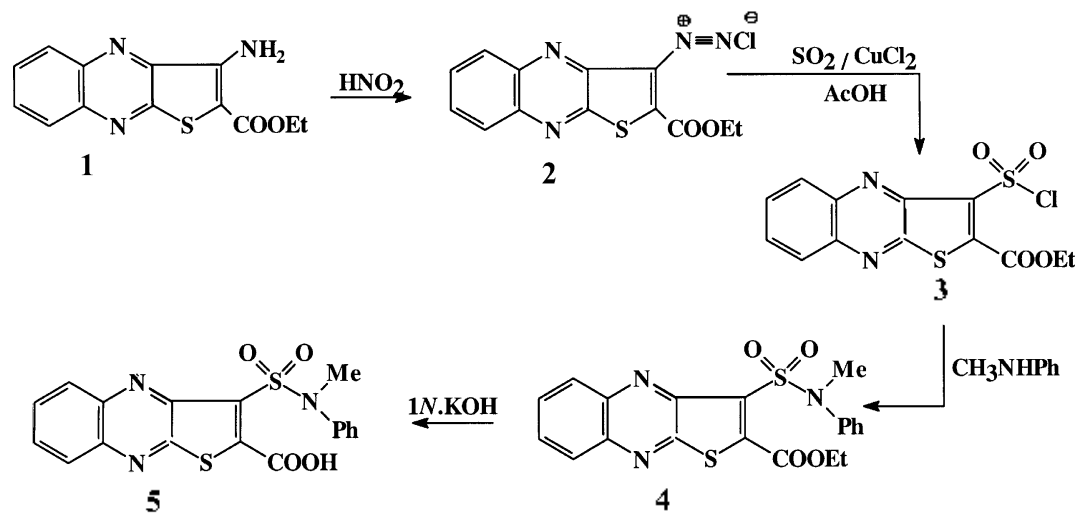

Scheme 1

'Corresponding Author: E-mail: oshmous(âaun.eun.eg 


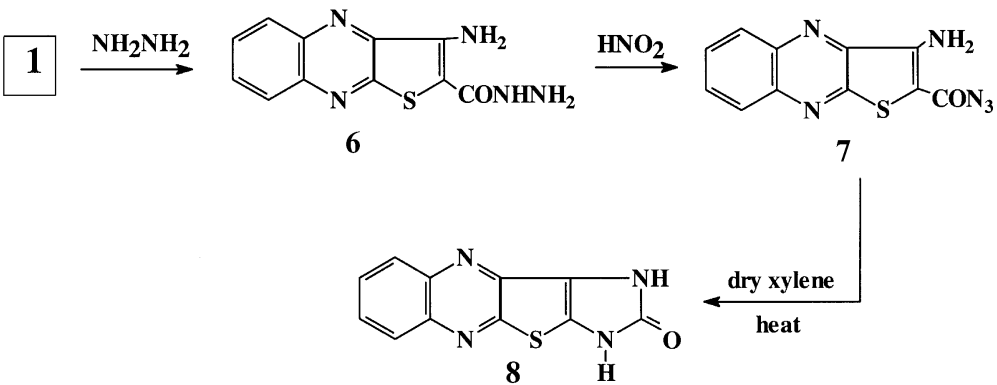

Scheme 2

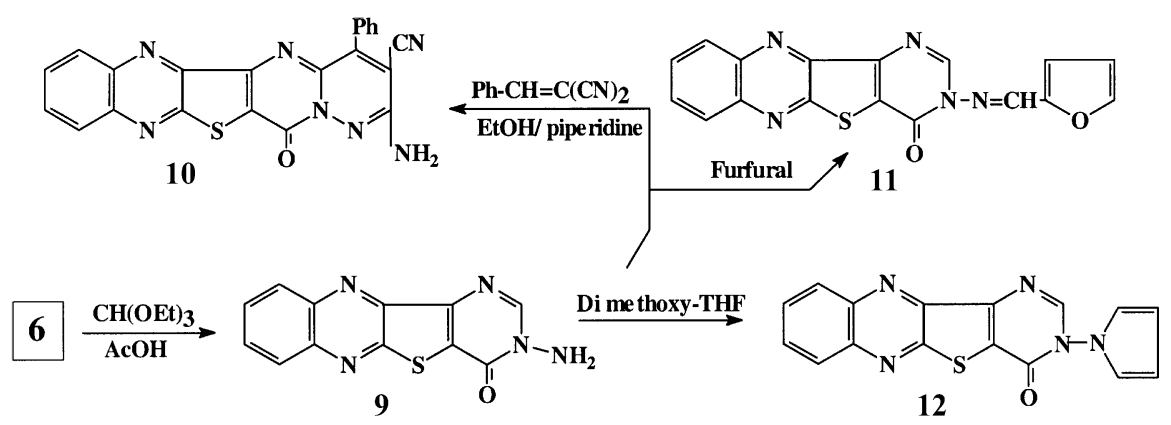

Scheme 3

zinopyrimidothieno[2.3-b] guinoxalinone $\mathbf{1 0}$. While the reaction of aminopyrimidine 9 with furfural and with dimethoxytetrahydrofuran provided the Schiff bases 11 and 3-(1pyrryl) derivative 12, respectively (Scheme 3).

When the carbohydrazide 6 was refluxed with carbon disulfide in pyridine gave oxadiazolylthienocuinoxaline $\mathbf{1 3}$ which was refluxed with hydrazine hydrate to produce 3amino-2-(2-hydrazino-1,3,4-oxadiazol-5-yl)thieno[2.3-b]quinoxaline 14. The latter compound was reacted with triethyl orthoformate and with nitrous acid to give (triazolo and tetrazolo)oxadiazolylthienoquinoxalines 15 and 16 resectively. Also, when $\mathbf{1 4}$ was allowed to react with carbon disulfide in pyridine the sulfanyltriazolooxadiazolyl derivative 17 was formed. Furthermore, the reaction of the thio- oxadiazol 13 with $\alpha$-halocompounds in the presence of sodium acetate yield S-alkylated derivatives 18 and 19 (Scheme 4).

\section{Experimental Section}

Melting points were determined on a Gallen Kamp melting point apparatus and were uncorrected. IR spectra were recorded on a Pye-Unicam $S \mathrm{P}^{3}-100$ spectrophotometer using $\mathrm{KBr}$ wafer technique. ${ }^{\mathrm{H}} \mathrm{H}$ NMR spectra were recorded on a $90 \mathrm{MHz}$. Varian T:M-390 NMR spectrometer in a suitable deutreated solvent (TMS) as the internal standard. Flemental analyses were determined on a Perkin-Elmer $240 \mathrm{C}$ microanalyzer. Elemental analysis, melting points, yields and

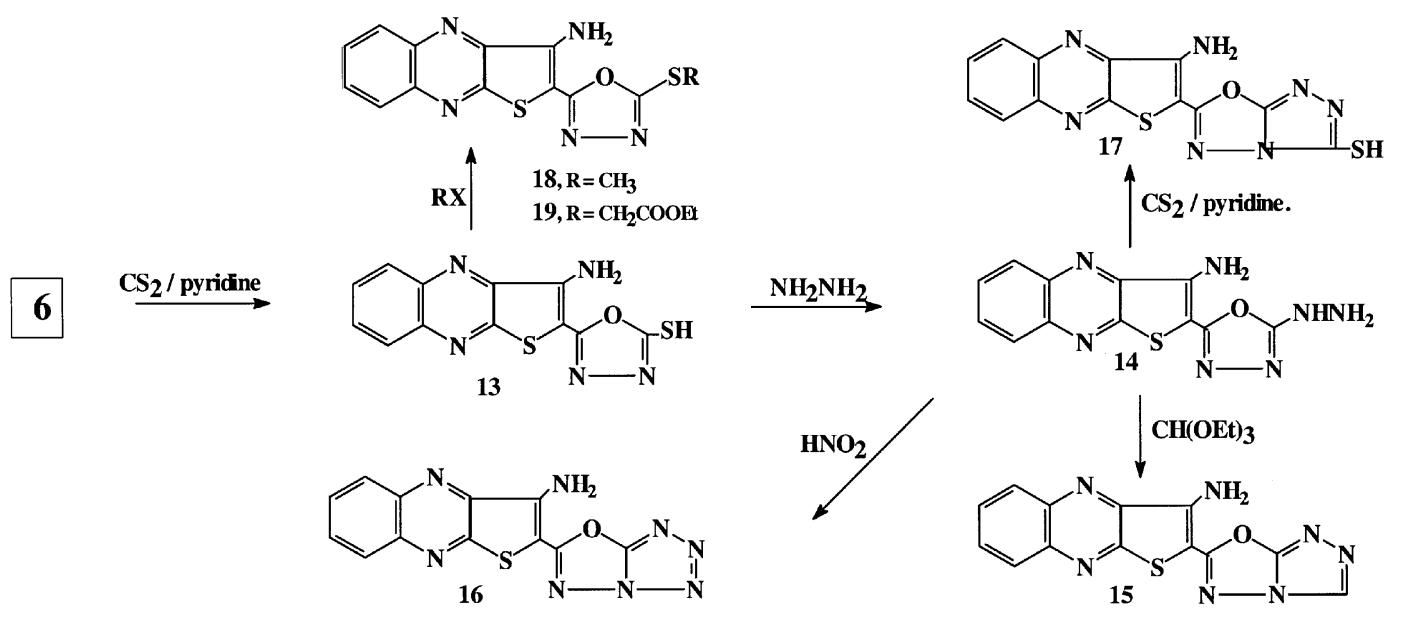

Scheme 4 
Table 1. Melting Points, Yields and Analytical Data of Compounds 3-19

\begin{tabular}{|c|c|c|c|c|c|c|}
\hline \multirow{2}{*}{$\begin{array}{c}\text { Comp. } \\
\text { No }\end{array}$} & \multirow{2}{*}{$\begin{array}{c}\text { M.P.P } \\
(\text { Yield\%) }\end{array}$} & \multirow{2}{*}{$\begin{array}{l}\text { Fonmula } \\
\text { Mol. Wt }\end{array}$} & \multicolumn{4}{|c|}{ Calculated/found } \\
\hline & & & $\mathrm{C}$ & $\mathrm{H}$ & $\mathrm{N}$ & $\mathrm{S}$ \\
\hline \multirow[t]{2}{*}{$3^{a}$} & 310 & $\mathrm{C}_{12} \mathrm{H}_{9} \mathrm{~N}_{2} \mathrm{O}_{4} \mathrm{~S}_{2} \mathrm{Cl}$ & 43.75 & 2.52 & 7.85 & 17.95 \\
\hline & $(80)$ & 356.5 & 43.67 & 2.41 & 7.79 & 17.89 \\
\hline \multirow[t]{2}{*}{4} & 180 & $\mathrm{C}_{20} \mathrm{H}_{1}: \mathrm{N}_{3} \mathrm{O}_{4} \mathrm{~S}_{2}$ & 56.20 & 3.98 & 9.83 & 14.98 \\
\hline & $(68)$ & 427 & 56.07 & 3.86 & 9.78 & 14.88 \\
\hline \multirow[t]{2}{*}{5} & $220-21$ & $\mathrm{C}_{13} \mathrm{H}_{13} \mathrm{~N}_{3} \mathrm{O}_{4} \mathrm{~S}_{2}$ & 54.13 & 3.25 & 10.52 & 16.04 \\
\hline & $(75)$ & 399 & 54.17 & 3.20 & 10.43 & 15.89 \\
\hline \multirow[t]{2}{*}{7} & 240 & $\mathrm{C}_{11} \mathrm{H}_{k} \mathrm{~N}_{6} \mathrm{OS}$ & 48.88 & 2.22 & 31.11 & 11.85 \\
\hline & $(83)$ & 270 & 48.79 & 2.18 & 31.07 & 11.78 \\
\hline \multirow[t]{2}{*}{8} & 190 & $\mathrm{C}_{13} \mathrm{H}_{k} \mathrm{~N}_{4} \mathrm{OS}$ & 54.54 & 2.47 & 23.14 & 13.22 \\
\hline & $(80)$ & 242 & 34.49 & 2.38 & 23.09 & 13.11 \\
\hline \multirow[t]{2}{*}{9} & 290 & $\mathrm{C}_{1} \mathrm{H}_{-} \mathrm{N}_{s} \mathrm{OS}$ & 53.53 & 2.60 & 26002 & 11.89 \\
\hline & $(70)$ & 269 & 53.44 & 2.56 & 26.12 & 11.90 \\
\hline \multirow[t]{2}{*}{10} & 225 & $\mathrm{C}_{\varkappa-\mathrm{H}_{11} \mathrm{~N}}=\mathrm{OS}$ & 62.70 & 2.61 & 23.27 & 7.60 \\
\hline & (77) & 421 & 62.61 & 2.54 & 23.00 & 7.34 \\
\hline \multirow[t]{2}{*}{11} & $>360$ & $\mathrm{C}_{1}: \mathrm{H}_{\circ} \mathrm{N}_{5} \mathrm{O}_{2} \mathrm{~S}$ & 58.78 & 2.59 & 20.17 & 9.22 \\
\hline & $(70)$ & 347 & 58.67 & 2.44 & 20.12 & 9.13 \\
\hline \multirow[t]{2}{*}{12} & 115 & $\mathrm{C}_{15} \mathrm{H}_{0} \mathrm{~N}_{5} \mathrm{OS}$ & 60.18 & 2.82 & 21.94 & 10.03 \\
\hline & $(90)$ & 319 & 59.98 & 2.77 & 21.84 & 10.00 \\
\hline \multirow[t]{2}{*}{13} & 320 & $\mathrm{C}_{12} \mathrm{H}_{7}-\mathrm{N}_{5} \mathrm{OS}_{2}$ & 47.84 & 2.32 & 23.25 & 21.26 \\
\hline & (81) & 301 & 47.76 & 2.23 & 23.14 & 21.19 \\
\hline \multirow[t]{2}{*}{14} & 260 & $\mathrm{C}_{12} \mathrm{H}_{0} \mathrm{~N}=\mathrm{OS}$ & 48.16 & 3.01 & 32.77 & 10.70 \\
\hline & (82) & 299 & 48.29 & 3.10 & 33.00 & 10.68 \\
\hline \multirow[t]{2}{*}{15} & $>360$ & $\mathrm{C}_{13} \mathrm{H}=\mathrm{N}=\mathrm{OS}$ & 50.48 & 2.26 & 31.71 & 10.35 \\
\hline & (75) & 309 & 50.30 & 2.22 & 31.61 & 10.27 \\
\hline \multirow[t]{2}{*}{16} & $>360$ & $\mathrm{C}_{1}: \mathrm{H}_{8} \mathrm{~N}_{8} \mathrm{OS}$ & 46.45 & 1.93 & 36.12 & 10.32 \\
\hline & $(69)$ & 310 & 46.50 & 1.90 & 36.21 & 10.36 \\
\hline \multirow[t]{2}{*}{17} & 120 & $\mathrm{C}_{13} \mathrm{H}=\mathrm{N}=\mathrm{OS}$ & 45.74 & 2.05 & 28.73 & 18.76 \\
\hline & $(90)$ & 341 & 45.61 & 2.00 & 28.66 & 18.59 \\
\hline \multirow[t]{2}{*}{18} & 150 & $\mathrm{C}_{13} \mathrm{H}_{4} \mathrm{~N}_{4} \mathrm{OS}$ & 49.52 & 2.85 & 22.22 & 20.31 \\
\hline & (78) & 315 & 49.60 & 2.86 & 22.16 & 20.22 \\
\hline \multirow[t]{2}{*}{19} & 240 & $\mathrm{C}_{1 \leqslant} \mathrm{H}_{1} \mathrm{~N}_{s} \mathrm{O}_{3} \mathrm{~S}_{2}$ & 49.61 & 3.35 & 18.08 & 16.33 \\
\hline & $(83)$ & 387 & 49.44 & 3.32 & 18.00 & 16.41 \\
\hline
\end{tabular}

${ }^{a} \mathrm{Cl}$ (calc. 9.95 . found $9.88^{\circ} \mathrm{i}$ ). spectroscopic data of compounds 3-19 are listed in Tables 1 and 2 .

3-Amino-2-ethoxycarbonylthieno[2,3-b]quinoxaline (1)

2-Ethoxycarbonylthieno[2,3-b]quinoxaline-3-diazonium chloride (2). Compounds 1 and 2 were prepared according to the literature. $\mathrm{mp} 145$ and $258^{\circ} \mathrm{C}$. respectively: Lit [15].

2-Ethoxycarbonylthieno[2,3-b]quinoxaline-3-sulfonylchloricle (3). To a solution of compound $2(0.01 \mathrm{~mol})$ in glacial acetic acid $(30 \mathrm{~mL})$ was added cupric chloride $(0.015$ mol). a slow rate of $\mathrm{SO}_{2}$ was passed through this solution for $\mathrm{l} \mathrm{lu}$, stand the reaction mixture for $2 \mathrm{~h}$ at RT and the mixture was poured into water. The solid separated was filtered and crystallized from acetic acid as greenish crystals.

Ethyl 3-(N-methyl-N-phenyl)sulfamoylthieno[2,3-b]quinoxaline-2-carboxylate (4). A mixture of $3(0.01 \mathrm{~mol})$ and $N$-methylaniline $(0.01 \mathrm{~mol})$ in absolute ethanol was refluxed for $3 \mathrm{~h}$, after cooling. The solid product thus formed was recrystallized from ethanol as red crystals.

3-(N-Methyl-N-phenyl)sulfamoylthieno[2,3-b]quinoxaline-2-carboxylic acid (5). A sample of $4(0.5 \mathrm{~g})$ in $10 \%$ $\mathrm{KOH}(25 \mathrm{~mL})$ was heated under reflux for $2 \mathrm{~h}$, then allowed to cool. neutralized with dilute $\mathrm{HCl}$. The solid product thus fomed was recrystallized from ethanol as red crystals.

3-Amino-2-thieno[2,3-b]quinoxaline-2-carboxylic acid hydrazide (6). Compound 6 was prepared according to the literature m.p. $305-306^{\circ} \mathrm{C}$ : Lit [14].

3-Amino-2-thieno[2,3-b]quinoxaline-2-carboazidle (7). To a misture of $6(0.01 \mathrm{~mol})$ and acetic acid $(15 \mathrm{~mL})$ was added sodium nitrite solution ( $14 \mathrm{~mL}, 0.01 \mathrm{~mol}$ ) at $0^{\circ}$ with stirring for $30 \mathrm{~min}$ and the resulting solid was filtered and recrystallized from ethanol as yellow crystals.

Imidazo[4',5':,+ 5$]$ thieno[2,3-b]quinoxalin-2(1H, 3H)-one (8). A sample of $7(0.5 \mathrm{~g})$ in dry xylene $(15 \mathrm{~mL})$ was heated under reflux for $30 \mathrm{~min}$, then allowed to cool. The solid product thus formed was recrystallized from ethanol as yellow crystals.

Table 2. Spectroscopic Data of Compounds 3-19

\begin{tabular}{|c|c|}
\hline $\begin{array}{l}\text { Compound } \\
\text { No. }\end{array}$ & $\operatorname{IR}\left(v \mathrm{~cm}^{-1}\right) /{ }^{\prime} \mathrm{H} \mathrm{NMR} \delta(\mathrm{ppm})$ \\
\hline 3 & $1720(\mathrm{CO}), 1620(\mathrm{C}=\mathrm{N}):\left(\mathrm{DMSO}-\mathrm{d}_{\mathrm{n}}\right): \delta 1.3-1.45\left(\mathrm{t}, 3 \mathrm{H}, \mathrm{CH}_{3}\right) 4.2-4.4\left(\mathrm{q}, 2 \mathrm{H}, \mathrm{CH}_{2}\right), 7.3-7.8(\mathrm{~mm}, 4 \mathrm{H}, \mathrm{Ar}-\mathrm{H})$ \\
\hline 4 & $1730(\mathrm{CO}), 1610(\mathrm{C}=\mathrm{N})(\mathrm{DMSO}-\mathrm{d}): 81.3-1.4(\mathrm{t}, 3 \mathrm{H}, \mathrm{CH}), 3.2\left(\mathrm{~s}, 3 \mathrm{H}_{3} \mathrm{CH}_{3}, 4.2-4.4\left(\mathrm{q}, 2 \mathrm{H}, \mathrm{CH}_{2}\right), 7.4-7.9(\mathrm{~m}, 9 \mathrm{H}, \mathrm{Ar}-\mathrm{H})\right.$ \\
\hline 5 & $1740(\mathrm{CO}), 1630(\mathrm{C}=\mathrm{N}) ;\left(\mathrm{CF}_{3} \mathrm{COOD}\right): \delta 3.2\left(\mathrm{~s}, 3 \mathrm{H}, \mathrm{CH}_{3}\right), 7,4-8.1(\mathrm{~m}, 9 \mathrm{H}, \mathrm{Ar}-\mathrm{H})$ \\
\hline 7 & $3380\left(\mathrm{NH}_{2}\right), 2180\left(\mathrm{~N}_{3}\right), 1730\left(\mathrm{~N}_{3}-\mathrm{CO}\right) ;\left(\mathrm{DMSO}-\mathrm{d}_{6}\right): \delta 6.2\left(\mathrm{~s}, 2 \mathrm{H}_{3} \mathrm{NH}_{2}\right), 7.5-8.0(\mathrm{~m}, 4 \mathrm{H}, \mathrm{Ar}-\mathrm{H})$ \\
\hline 8 & $3210,3185(\mathrm{NH}), 1670(\mathrm{CO})\left(\mathrm{CDCl}_{3}\right): 87.5-8.3(\mathrm{~m}, 4 \mathrm{H}, \mathrm{Ar}-\mathrm{H}), 9.2,10.3(\mathrm{~s}, 2 \mathrm{H}, \mathrm{NH})$ \\
\hline 9 & $3340\left(\mathrm{NH}_{*}\right), 1680(\mathrm{C}=\mathrm{O}),\left(\mathrm{DMSO}-\mathrm{d}_{\mathrm{r}}\right): \delta 6.3(\mathrm{~s}, 2 \mathrm{H}, \mathrm{NH}), 7.5-7.8(\mathrm{~m}, 4 \mathrm{H}, \mathrm{Ar}-\mathrm{H}), 9.5(\mathrm{~s}, \mathrm{lH}, \mathrm{CH})$ \\
\hline 10 & $3420\left(\mathrm{NH}_{*}\right), 2220(\mathrm{CN}), 1700(\mathrm{C}=\mathrm{O}) ;\left(\mathrm{DMSO}-\mathrm{d}_{\mathrm{r}}\right): 86.2(\mathrm{~s}, 2 \mathrm{H}, \mathrm{NH}), 7.4-7.9(\mathrm{~m}, 9 \mathrm{H}, \mathrm{Ar}-\mathrm{H})$ \\
\hline 11 & $2960(\mathrm{CH}$, aliph. $), 1690(\mathrm{CO}), 1620(\mathrm{C}=\mathrm{N})$. \\
\hline 12 & $1700(\mathrm{CO}) 1640(\mathrm{C}=\mathrm{N}) ;(\mathrm{DMSO}-\mathrm{d}): \delta 6.1,6.3(2 \mathrm{~d}, 4 \mathrm{H}, \mathrm{CH}-\mathrm{pyrrole}), 7.6-8.2(\mathrm{~m}, 4 \mathrm{H}, \mathrm{Ar}-\mathrm{H}), 9.4(\mathrm{~s}, 1 \mathrm{H}, \mathrm{CH})$. \\
\hline 13 & $3430\left(\mathrm{NH}_{*}\right), 1230,1060(\mathrm{SH}) ;\left(\mathrm{DMSO}-\mathrm{d}_{6}\right): \delta 6.24\left(\mathrm{~s}, 2 \mathrm{H}, \mathrm{NH}_{2}\right), 7.5-8.2(\mathrm{~m}, 4 \mathrm{H}, \mathrm{Ar}-\mathrm{H})$ \\
\hline 14 & $3220,3500\left(\mathrm{NH}, \mathrm{NH}_{2}\right), 1630(\mathrm{C}=\mathrm{N}) ;\left(\mathrm{CF}_{3} \mathrm{COOD}\right): 87.5-7.95(\mathrm{~m}, 4 \mathrm{H}, \mathrm{Ar}-\mathrm{H})$ \\
\hline 15 & $3430\left(\mathrm{NH}_{*}\right),\left(\mathrm{DMSO}-\mathrm{d}_{6}\right): \delta 6.15\left(\mathrm{~s}, 2 \mathrm{H}, \mathrm{NH}_{*}\right), 7.5-8.2(\mathrm{~m}, 4 \mathrm{H}, \mathrm{Ar}-\mathrm{H}), 9.4\left(\mathrm{~s}, 1 \mathrm{H}_{3} \mathrm{CH}\right)$ \\
\hline 16 & $3400\left(\mathrm{NH}_{2}\right), 1620(\mathrm{C}=\mathrm{N}) ;\left(\mathrm{CF}_{3} \mathrm{COOD}\right): 87.5-7.95(\mathrm{~m}, 4 \mathrm{H}, \mathrm{Ar}-\mathrm{H})$ \\
\hline 17 & $3400\left(\mathrm{NH}_{*}\right), 1220,1050(\mathrm{SH}) ;\left(\mathrm{DMSO}-\mathrm{d}_{6}\right): \delta 6.25\left(\mathrm{~s}, 2 \mathrm{H}, \mathrm{NH}_{2}\right), 7.45-8.0(\mathrm{~m}, 4 \mathrm{H}, \mathrm{Ar}-\mathrm{H})$ \\
\hline 18 & $3410\left(\mathrm{NH}_{*}\right):\left(\mathrm{CDCl}_{3}\right): \delta 3.3\left(\mathrm{~s}, 3 \mathrm{H}, \mathrm{CH}_{3}\right), 6.1\left(\mathrm{~s}, 2 \mathrm{H}, \mathrm{NH}_{*}\right) 7.6-8.2\left(\mathrm{~m}_{3}, 4 \mathrm{H}, \mathrm{Ar}-\mathrm{H}\right)$ \\
\hline 19 & $3420\left(\mathrm{NH}_{2}\right), 2930(\mathrm{CH}, \mathrm{aliph}), 1720(\mathrm{C}=\mathrm{O}) ;(\mathrm{CDCl}): \delta 1.4-\mathrm{I} .6\left(\mathrm{t}, 3 \mathrm{H}, \mathrm{CH}_{3}\right), 4.1-25\left(\mathrm{q}, 2 \mathrm{H}, \mathrm{CH}_{2}\right), 6.2\left(\mathrm{~s}, 2 \mathrm{H}, \mathrm{NH}_{2}\right), 7.4-8.1(\mathrm{ml}, 4 \mathrm{H}, \mathrm{Ar}-\mathrm{H})$ \\
\hline
\end{tabular}


3-Amino-pyrimido $\left[4^{\prime}, 5^{\prime}: 4,5\right]$ thieno[2,3-b] quinoxalin- $+(2 \mathrm{H})$ one (9). To a mixture of $6(0.015 \mathrm{~mol})$ and triethyl orthoformate $(5 \mathrm{~mL})$ in methanol $(30 \mathrm{~mL})$, few drops of acetic acid were added. The mixture was heated under reflux for 5 $h$, then allowed to cool. The solid product thus formed was recry stallized from ethanol as red crystals.

2-Amino-3-cyano-4-Phenylpyridazino[2", 3': 1',2']pyrimido $\left[4^{\prime}, \mathbf{5}^{\prime}: 4,5\right]$ thieno[2,3-b]quinoxalin-13-one (10). A solution of $9(0.01 \mathrm{~mol})$. arylidene malonodinitrile $(0.01$ $\mathrm{mol})$ in absolute ethanol $(10 \mathrm{~mL})$ and $(1 \mathrm{~mL})$ of triethylamine. was stirred at reflux for $2 \mathrm{~h}$. After cooling the solid product was filtered, washed with cool ethanol and recry stallized from ethanol as pale yellow crystals.

3-(2-Furfurilidene)aminopyrimido $[4,5,: 4,5]$ thieno $[2,3-$ b]quinoxalin-4(3H)-one (11). A solution of compound 9 $(0.01 \mathrm{~mol})$ and furfural $(0.015 \mathrm{~mol})$ in glacial acetic acid $(40$ $\mathrm{mL}$ ) was heated at $80^{\circ}$ for $4 \mathrm{~h}$. After cooling the separated solid was filtered and crystallized from acetic acid as browinsh crystals.

3-(1-Pyrryl)pyrimido[4',5':4,5] thieno[2,3-b]quinoxalin$4(3 \mathrm{H})$-one (12). To a solution of compound $9(0.01 \mathrm{~mol})$ in glacial acetic acid $(20 \mathrm{~mL})$ was added dimethoxy-tetrahydrofuran $(0.015 \mathrm{~mol})$. and the mixture was refluxed, stirred for $2 \mathrm{~h}$. evaporated under redused pressure, the residue was crystallized from ethanol/acetic acid as redish crystals.

3-Amino-2-(5-sulfanyl-1,3,4-0xadiazol-2-y])thieno[2,3b]quinoxaline (13). A nixture of $6(0.01 \mathrm{~mol})$ and carbon disulfide $(5 \mathrm{~mL})$ in pyridene $(25 \mathrm{~mL})$ was refluxed on steam bath for $8 \mathrm{~h}$. then allowed to cool. The solid product thus formed was recrystallized from ethanol as red crystals.

3-Amino-2-(5-hydrazino-1,3,t-oxadiazol-2-yl)thieno[2,3b] quinoxaline (14). A mixture of $13(0.01 \mathrm{~mol})$ and hydrazine hydrate $(6 \mathrm{~mL})$ was refluxed in ethanol $(35 \mathrm{~mL})$ for $(5$ $h$, or until evoluation of $\mathrm{H}_{\mathrm{S}} \mathrm{S}$ cease) then allowed to cool, the yellow precipitate was filtered off and recrystallized from ethanol.

3-Amino-2-(1,2,4-triazolo[3,4-b][1,3,4]oxadiazol-6-y])thieno[2,3-b]quinoxaline (15). To a mixture of $14(0.015$ $\mathrm{mol})$ and triethyl orthoformate $(3 \mathrm{~mL})$ in methanol $(20 \mathrm{~mL})$. few drops of acetic acid were added. The mixture was heated under reflux for $3 \mathrm{~h}$, then allowed to cool. The solid product thus formed was recrystallized from acetic acid as pale red crystals.

3-Amino-2-(tetrazolo[4,5-b][1,3,4] oxadiazol-6-yl)thieno[2,3-b]quinoxaline (16). To a solution of $1+(0.01 \mathrm{~mol})$ in acetic acid $(25 \mathrm{~mL})$ was added dropwise sodium nitrite solution $(14 \mathrm{~mL} .0 .01 \mathrm{~mol})$ at 0 with stirring for $2 \mathrm{~h}$. The resulting solid was filtered and recrystallized from ethanol as yellowish crystals.

3-Amino-2-(3-sulfanyl-1,2,t-triazolo[3,t-b]oxadiazo]6-yl)thieno[2,3-b]quinoxaline (17). A mixture of $1+(0.01$ mol) and carbon disulfide $(5 \mathrm{~mL})$ in pyridine $(35 \mathrm{~mL})$ was refluxed on steam bath for $6 \mathrm{~h}$. then allowed to cool. The solid product thus formed was recrystallized from acetic acid as pale red crystals.

3-Amino-2-(5-methylthio-1,3,4-oxadiazol-2-yl)thieno[2,3b]quinoxaline (18). A mixture of 13 (0.01 mol). methyl iodide $(0.01 \mathrm{~mol})$ and anhydrous sodium acetate $(5 \mathrm{~g}) \mathrm{in}$ ethanol $(40 \mathrm{~mL})$ was refluxed for $2 \mathrm{~h}$. poured onto cold water. The solid obtained was filtered off and recrystallized from ethanol as pale yellow crystals.

3-Amino-2-(5-ethylacetatethio-1,3,t-oxadiazol-2-yl)thieno[2,3-b]quinoxaline (19). A mixture of $13(0.0 \mathrm{l}$ mol). ethyl chloroacetate $(0.01 \mathrm{~mol})$ and anhydrous sodium acetate $(5 \mathrm{~g})$ in ethanol $(30 \mathrm{~mL})$ was refluxed for $2 \mathrm{~h}$, poured onto cold water. The solid obtained was filtered off and recrystallized from ethanol as pale yellow crystals.

\section{References}

1. Zhu. Z.: Saluja. S.: Drach. C. J.: Townsend. L. P. J. of the Chinese Chem. Soc. 1988, 45(4). 465 .

2. (a) Makino. K. Kim. H. S.: Yoshihisa. K. J. Heterocyel. Chem. 1998. 35. 321. (b) Makino, K.: Kim. H. S.: Yoshihisa, K. $d$. Heterocycl. Chem. 1998. 35. 489 .

3. Sainz. Y.: Montoya. M. E.: Martinez. F. J.: Ortega. M. A.: Lopez. A.: Monge. A. Arznient. Forsch 1999. 49. 55. [Chent Abst 1999. $130,3827 \mathrm{P}$.

4. Mange, A.: Palop, J. A.; Urbasos. I.: Femandez-Alvarez, E. $d$. Heterocyct Chem $1989,26.1623$

5. Yoo. H. W. Yur1-Sil. L.: Sul. M. E.: Kiml. D. J.: Park. S. W. Arch Pham 1998. 331.10331.

6. Gozyo. S.: Kenzi. M.: Yoshihisa. K. Heterocycles 1988. 27. 2481.

7. Kaneko. C.: Katagiri, S. (Asahi Glass Co.Ltd), Japan Kokai Tokkyo Koho Jp. (patent) 1988. 62, 207, 264. [Chem. Abstr. 1988. 109.231061f].

8. Hiramatsu. T.: Azuma. S.: Nakagawa. K.: Ichikawa. Y. (Teijit1 Ltd.) Japan Kokai Tokkyo Koho Jp. (patent). 1988. 62. 163. 263. [Chem. Atstr 1988. 109.73473k].

9. Miguel. A. O.: Maria, J. M.: Francisco. J. M.: Yolanda, S.: Maria, E. M.: Adele, L. C.: Antonio, M. Eur J. Wed. Chem Chim Ther: 2000. 35,121

10. El-Ashry. E. S. H.: Abdel-Rahmanl. A. A. H.: Rashed. N.: Rasheed. H. A. Phomazie 1999. $5+(12) .893$.

11. El-Hawash. S. A.: Habib. N. S.: Fanaki. N. H. Phornazie 1999. 54. 808.

12. Bindumhavan. V: Prabhakar, B. C.; Kumar. C. D.; John, D. N:; Helmut. R. R. (Hoechst India Ltd.) Indian IN 167,425 (patent) 1993. [Chem. Abstr. 1993. 119. 117279q].

13. Moustafa. O. S. Phosphonts, Sulfu and Sificon 1997. 131. 49.

14. Badr. M. Z. A.: Mahgoub. S. A.: Moustafa. O. S. J. Chent. Soc. Pakestan 1993. 15. 264.

15. Badr. M. Z. A.; Mahgoub, S. A.: Moustafa. O. S. Phosphorus Sulfur and Silicon 1993, 79. 477.

16. Moustafa. O. S. Phosphonts, Suffir and Silicon 1999.155. 235.

17. Moustafa. O. S. J of the Chinese Chem. Soc. 2000. 47(2). 351.

18. Geies. A. A.: El-Deen. A. M. K.: Moustafa. O. S. Pharmazie 1996. $52(6), 437$

19. Moustafa. O. S.; Badr. M. Z. A.; Kamel. E. M. Phammazie 2000. $55(12) .896$ 